\title{
Gastrointestinale Erkrankungen: Forschungsschwerpunkte der Pharmafirmen
}

\section{Virushepatitiden}

Seit der Einführung von oralen, gut verträglichen und sehr effektiven Therapien, die auf direkt wirkenden antiviralen Agenzien beruhen, hat sich die Behandlung der Hepatitis C dramatisch verändert. Einst eine chronische Erkrankung, für die im Gegensatz zur Hepatitis A und B keine Impfung zur Verfügung steht und die nur in einem Teil der Patienten mit einer langen, nebenwirkungsreichen Therapie behandelbar war, kann eine Hepatitis-C-Virusinfektion heute in 8-12 Wochen nahezu bei allen Patienten mit einmal täglicher Gabe von 1-3 Tabletten geheilt werden [1]. Mit diesem therapeutischen Durchbruch scheint eine weitere Verbesserung der Regimes in naher Zukunft nicht absehbar. Diese hochwirksamen Therapien bilden zusammen mit dem Addendum der S3Leitlinie, welche eine Behandlung aller Patienten mit einer replikativen HCV-Infektion empfiehlt, und der Aufnahme des Hepatitis-C-Screenings innerhalb der Gesundheitsuntersuchung (Check up 35) ab 1. Oktober 2021 [2] die ideale Voraussetzung für eine HCV-Elimination.

\section{Chronisch-entzündliche Darmerkrankungen (CED)}

Ein zentraler Forschungsschwerpunkt der pharmazeutischen Industrie bleiben chronisch-entzündliche Darmerkrankungen. Darüber hinaus wird in geringerem Umfang an Zöliakie und gastrointestinale Motilitätsstörungen geforscht. CED umfassen ein Spektrum immunvermittelter entzündlicher Erkrankungen mit komplexer multifaktorieller Pathogenese und äußerst heterogener, patientenindividueller klinischer Ausprägung. Diese Komplexität wird höchstwahrscheinlich eine Reihe von Medikamenten erfordern, die mit unterschiedlichen Ansätzen darauf abzielen, eine steroidfreie anhaltende Remission und Mukosaheilung zu erreichen.

Mit den derzeit verfügbaren Therapien wird bei $20-30 \%$ der Patienten ein primäres Nichtansprechen beobachtet, und weitere $30 \%$ der Patienten werden aufgrund eines sekundären Ansprechverlustes refraktär [3]. Diese Zahlen spiegeln den „unmet need“ der Krankheitskontrolle wider und belegen eindrücklich die Notwendigkeit der Entwicklung neuer zielgerichteter Therapeutika mit hoher Effektivität und Sicherheit.

Derzeit basiert die medizinische Behandlung der CED hauptsächlich auf 5-Aminosalicylaten, Kortikosteroiden, Thiopurinen, Anti-TNF-Inhibitoren, einer Anti-Integrinoder einer Anti-Interleukin-(IL)-12/IL-23Therapie. Neue Erkenntnisse zu den zugrunde liegenden Mechanismen der Pathogenese der CED haben Fortschritte bei der Entwicklung neuer Behandlungsoptionen ermöglicht. Die Januskinasen (JAK)-Inhibitoren zielen auf verschiedene JAK/ STAT-vermittelte Signalwege ab, die an der Pathogenese der CED beteiligt sind. Tofacitinib, ein JAK-Inhibitor, der hauptsächlich auf JAK1 und JAK3 abzielt, wurde für die Behandlung der Colitis ulcerosa (CU) zugelassen. Selektive JAK1-Inhibitoren befinden sich derzeit in der Entwicklung und haben bei CED in Phase-3-Studien hohe Effektivitätsraten gezeigt (Filgotinib [4] und Upadacitinib [5]). Zudem steht voraussichtlich bald ein Sphingosin-1-phosphat-Rezeptor (S1PR)-Agonist (Ozanimod) bei Colitis ulcerosa zur Verfügung. Diese Substanz wirkt antiinflammatorisch, indem der Austritt der Lymphozyten aus den Lymphknoten gehemmt wird. Risankizumab und Mirikizumab sind Biologika in der späten Phase der Entwicklung bei Morbus Crohn und Colitis ulcerosa, die selektiv und mit hoher Affinität an die p19-Untereinheit des humanen Interleukins 23 binden, jedoch nicht an IL-12. IL-23 ist ein regulatorisches Zytokin, das unter anderem die Bildung, den Erhalt und die Aktivierung von Typ-17-T-Helferzellen (Th17) fördert. Diese produzieren proinflammatorische Zytokine wie IL-17A und IL-22, die eine wichtige Rolle bei chronischen Entzündungsprozessen spielen. Durch die Rezeptorblockade werden die IL-23-abhängige Signalübertragung und die Freisetzung dieser Botenstoffe gehemmt.

Unabhängig von der aktuellen oder zukünftigen Wahl der individuellen Therapie des Patienten gilt: In jedem Fall sind eine regelmäßige Verlaufskontrolle und das Verfolgen strikter Therapieziele notwendig, bei denen subjektive (Symptome) und objektive Parameter (wie endoskopische Verbesserung) der Krankheitsausprägung in die Betrachtung einbezogen werden sollten.

In Erwartung neuer Therapien mit möglicherweise besserer Effektivität werden neue ambitionierte Therapieziele bei CED diskutiert, die langfristig den Krankheitsverlauf positiv beeinflussen könnten. Im Rahmen der Initiative STRIDE (Selecting Therapeutic Targets in Inflammatory Bowel Disease) hat ein Expertenkomitee der International Organization for the Study of IBD (IOIBD) objektiv nachverfolgbare Ziele für die Therapie von CED formuliert. Die erstmals im Jahr 2015 veröffentlichten Empfehlungen (STRIDE-I) [6] wurden kürzlich überarbeitet. Aufbauend auf die erste Fassung orientieren sich die Ziele auch weiterhin an der „Treat to Target“-Strategie. Die nun in STRIDE-II formulierten Ziele für die CED-Therapie spiegeln den evidenzbasierten Expertenkonsens der beteiligten IOIBD-Mitglieder wider [7]. Ausgangsbasis für das Update der Empfehlungen waren einerseits eine systematische Literaturrecherche und andererseits mehrere Frage- und Abstimmungsrunden, in denen die Experten die aus ihrer Sicht wichtigsten Therapieziele definiert haben.

STRIDE-II umfasst insgesamt 13 neue oder überarbeitete Empfehlungen für die verschiedenen Phasen des Therapieverlaufs. Die Autoren weisen darauf hin, dass die Zeitspanne, in der erwartungsgemäß ein klinisches Ansprechen, eine klinische Remission oder eine endoskopische Heilung erreicht werden können, bei den verschiedenen CED-Therapien variiert.

Initial ist als Ziel definiert, zunächst ein rasches klinisches Ansprechen zu erreichen. Mittelfristig sollen klinische Remission und die Normalisierung des fäkalen Entzündungsmarkers Calprotectin in einen akzeptablen Bereich sowie die Normalisierung des C-reaktiven Proteins erreicht werden. Langfristige Therapieziele sind endoskopische Heilung, die Wiederherstellung der Lebensqualität und die Ver- 
meidung von irreversiblen CED-bedingten Einschränkungen bzw. Behinderungen.

Als langfristiges wichtiges Kriterium, wenngleich noch nicht als formales Therapieziel, wurden außerdem die transmurale Heilung (Morbus Crohn) sowie die histologische Heilung (Colitis ulcerosa) in die STRIDE-II-Empfehlungen aufgenommen, da sie eine tiefere Heilung reflektieren und möglicherweise einen prognostisch günstigen Krankheitsverlauf erwarten lassen.

In einer Studie aus dem Jahr 2019 konnte beispielsweise gezeigt werden, dass das Erreichen einer histologischen Remission eindeutig einen prognostischen Wert hat für die Wahrscheinlichkeit, eine langfristige klinische Remission (über 5 Jahre) zu erhalten im Vergleich zu den Patienten, die „nur" eine endoskopische Heilung erreicht haben [8]. Allerdings konnte hier nur bei etwa 15\% der Patienten die histologische Heilung überhaupt erzielt werden.

In der Entwicklung befindliche, zielgerichtete Therapien adressieren in den Zulassungsstudien strenge Endpunkte wie komplette endoskopische oder histologische Heilung sowie die Kombination aus beidem. Inwieweit können die neuen, zielgerichteten Therapien, ggf. auch die Kombinationen aus diesen, in Zukunft bei selektierten Patienten nachhaltig den Krankheitsverlauf positiv beeinflussen? Das wird Gegenstand zukünftiger Untersuchungen sein.

Bei den kürzlich vorgestellten ersten Ergebnissen des Phase 3-Programms mit dem JAK-Inhibitor Upadacitinib erreichten bis zu 44\% der im Mittel mehr als 8 Jahre an Colitis ulcerosa erkrankten Patienten bereits zur Woche 8 eine endoskopische Verbesserung (endoskopischer Mayo-Subscore $\leq 1)[9,10]$. Darüber hinaus konnte bei bis zu $49 \%$ der Patienten eine „HEMI“
(= histologisch-endoskopische mukosale Verbesserung) im Vergleich zu 12\% der Patienten in der Placebogruppe $(p<0,001)$ nach 52 Wochen erzielt werden [11].

Der endoskopischen und histologischen Evaluation des Darmes kommt somit in den klinischen Studien mit neueren Biologika- und JAK-Therapieansätzen eine große Bedeutung zu. Denn einerseits belegen sie objektiv die „antiinflammatorische Potenz" neuartiger zielgerichteter Therapien und geben zudem Hoffnung, ambitioniertere Therapieziele mit prognostischer Bedeutung für den Krankheitsverlauf zu erreichen.

\section{Interessenkonflikt}

Björn Fritz ist Mitarbeiter der AbbVie Deutschland $\mathrm{GmbH}$ \& Co. KG und besitzt möglicherweise AbbVie-Aktien. AbbVie war an der Erstellung dieses Abstracts beteiligt.

\section{Autor}

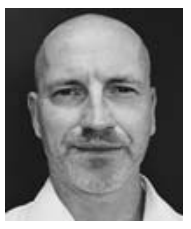

\section{Björn Fritz}

Dr., Medical Director, AbbVie Deutschland GmbH \& Co. KG, Wiesbaden, Deutschland

\section{Korrespondenzadresse}

\section{Dr. Björn Fritz}

Medical Director

AbbVie Deutschland GmbH \& Co. KG

Mainzer Straße 81

65189 Wiesbaden

Deutschland

bjoern.fritz@abbvie.com

\section{Literatur}

[1] The hunt for a vaccine for hepatitis $C$ virus continues. Lancet Gastroenterol Hepatol 2021; 6: 253

[2] Institut des Bewertungsausschusses. Beschluss des Bewertungsausschusses nach
$\S 87$ Abs. 1 Satz 1 SGB V in seiner 567. Sitzung am 04.08.2021. Im Internet (Stand: 05.10.2021): https://institut-ba.de/ba/babeschluesse/2021-08-04_ba567_6.pdf

[3] Sabino ], Verstockt B, Vermeire $S$ et al. New biologics and small molecules in inflammatory bowel disease: an update. Therap Adv Gastroenterol 2019; 12: 1756284819853208. doi:10.1177/1756284819853208

[4] Feagan BG, Danese S, Loftus Jr. E et al. Filgotinib as induction and maintenance therapy for ulcerative colitis (SELECTION): a phase 2b/3 double-blind, randomised, placebocontrolled trial. Lancet 2021; 397: 23722384

[5] Im Internet (Stand: 05.10.2021): https:// news.abbvie.com/news/press-releases/second-phase-3-induction-study-confirmsupadacitinib-rinvoq-improved-clinical-endoscopic-and-histologic-outcomes-in-ulcerative-colitis-patients.htm

[6] Peyrin-Biroulet L, Sandborn W, Sands BE et al. Selecting therapeutic targets in inflammatory bowel disease (STRIDE): determining therapeutic goals for treat-to-target. Am J Gastroenterol 2015; 110: 1324-1338

[7] Turner D, Ricciuto A, Lewis A et al. STRIDE-II: an update on the Selecting Therapeutic Targets in Inflammatory Bowel Disease (STRIDE) Initiative of the International Organization for the Study of IBD (IOIBD): Determining therapeutic goals for treat-to-target strategies in IBD. Gastroenterology 2021; 160: 1570-1583

[8] Christensen B, Erlich J, Gibson PR et al. Histologic healing is more strongly associated with clinical outcomes in ileal Crohn's disease than endoscopic healing. Clinical Gastroenter Hepatol 2020; 18: 2518-2525

[9] Im Internet: https://news.abbvie.com/news/ press-releases/second-phase-3-inductionstudy-confirms-upadacitinib-rinvoq-improved-clinical-endoscopic-and-histologic-outcomes-in-ulcerative-colitis-patients.htm

[10] Im Internet: https://news.abbvie.com/alerttopics/immunology/upadacitinib-rinvoqmeets-primary-and-all-ranked-secondaryendpoints-in-first-phase-3-induction-studyin-ulcerative-colitis.htm

[11] Im Internet: https://news.abbvie.com/news/ press-releases/upadacitinib-rinvoq-met-primary-and-all-secondary-endpoints-in-52week-phase-3-maintenance-study-in-ulcerative-colitis-patients.htm 